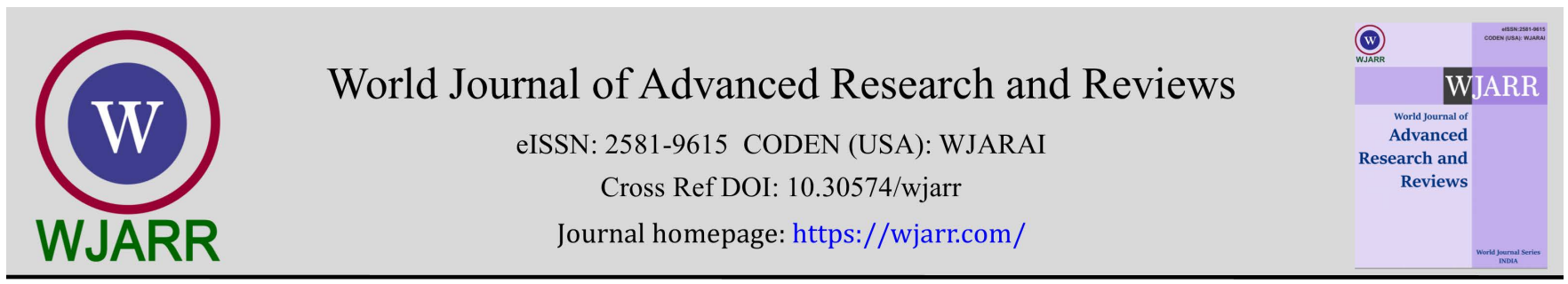

(REVIEW ARTICLE)

\title{
Neurocysticercosis: A rare disorder
}

\author{
Diya Wilson ${ }^{1}$ and Khayati Moudgil 2,* \\ ${ }^{1}$ PharmD Intern, Department of Pharmacy Practice, JSS College of Pharmacy, Ooty, India. \\ ${ }^{2}$ Faculty of Health Sciences, School of Pharmacy, JSS Academy of Higher Education and Research, Mauritius.
}

World Journal of Advanced Research and Reviews, 2021, 09(01), 286-291

Publication history: Received on 17 January 2021; revised on 24 January 2021; accepted on 26 January 2021

Article DOI: https://doi.org/10.30574/wjarr.2021.9.1.0030

\begin{abstract}
Neurocysticercosis (NCC) is a rare central nervous system infection that is preventable and is caused by the Taenia solium tapeworm larval stage. This disorder is the world's most common cause of acquired epilepsy and a significant cause of neurological morbidity. This disease is pleomorphic due to several complex factors such as cysticerci characteristics, stages of development, and many more which leads to difficulties in inaccurate diagnosis and adequate stage-wise care. The introduction of cystidical medications, however, has led to a stronger prognosis of NCC-affected patients with an increase in the patient's clinical course of the disease. After diagnosis most seizure patients do not experience epilepsy. Since Neurocysticercosis remains a neglected tropical disease, the management, and prevention of T. so ilium transmission and strategies for eradicating NCC from endemic areas should be highlighted and prioritized in global healthcare to minimize the significant burden on healthcare and the economy.
\end{abstract}

Keywords: Neurocysticercosis; Healthcare; Tropical; Neglected

\section{Introduction}

Neurocysticercosis is a severe, and preventable, helminthic infection of the central nervous system that is prevalent in most developing countries. The World Health Organization (WHO) has declared the disease a "Neglected Tropical Disease" Caused by Taenia Solium, commonly known as the pork tapeworm, the larval stage of the parasite is responsible for the introduction of the disease in humans, which then contributes to the production of symptoms in different organ tissues, which may vary depending on the position and number of cysts. The reason NCC is endemic owes to conditions promoting parasite transmissions such as poor hygiene \& sanitation, and factors such as deprivation \& analphabetism. Yet the expansion of NCC cannot be limited exclusively to developing countries like Latin America, Saharan regions, and Asia. High-income countries like North America, European regions have also been found to be suffering from the significant burden of mortality and morbidity due to increased immigration from endemic areas. This disease is a very interesting one because of the host's unpredictable immunological response to the cysticerci and also because of its heterogeneity. It is therefore very important to precisely identify the position of the parasite in the CNS and to differentiate between parenchymal which extra-parenchymal NCC and is a very important step because these are two distinct entities with discrepancies in immunology and pathophysiology aspects. Therefore, diagnosing correctly and having a prognosis is strenuous for health care providers. While modern diagnostic tools and treatment strategies are available for managing this disease; interestingly, there is a lack of investment specifically in endemic regions in cost-effective methods of eradication. Here, the analysis focuses on various features of this disease with a focused perspective on its epidemiology, pathophysiology, and obtains an unbiased view of current treatment aspects and methods of prevention.

\footnotetext{
${ }^{*}$ Corresponding author: Dr. Khayati Moudgil

Faculty of Health Sciences, School of Pharmacy, JSS Academy of Higher Education and Research, Mauritius. 


\section{Epidemiological findings}

The World Health Organization reports that about 50 million people worldwide have Neurocysticercosis and that this disorder causes about 50,000 deaths per year. It has been found that NCC is also responsible in resistant regions for more than 50 percent of late-onset epilepsy cases. Most developing countries in the Sub-Saharan, Latin American, and Asian regions are endemic hotspots that give rise to the worldwide surplus manifestation of this infection. Just as industrialization, improved husbandry practices, and primitive healthcare have contributed to the virtual absence of $\mathrm{T}$. solium from developing countries, cysticercosis and neurocysticercosis are again diagnosed in North America, Europe, and Australia as a result of increased immigration from endemic areas [1]. While Neurocysticercosis tends to affect men and women alike, there is some evidence indicating that inflammation may be more intense in women than in men around the cysts. But several observational studies have shown that the prevalence of NCC in both sexes is not substantially different. Gender cannot, therefore, be considered a risk factor [2]. Furthermore, it is a fact that Neurocysticercosis tends to be the most common cause of seizures in children and adults (peak incidence, 30-40 y), the exact incidence in children is not understood, and not many studies in this population have been performed. India, as a developing country, lacks adequate diagnostic facilities, and especially the management of these infectious diseases in rural areas. Based on the findings of a community-based study conducted in Tamilnadu district in southern India involving more than 50,000 individuals, NCC was found to be the cause of active epilepsy in at least $1 / 3 \mathrm{rd}$ of the patients involved in the studies (at least 1 seizure episode in the five years preceding the survey) and thus the prevalence of NCC in India was determined to be one per thousand population in India [3]. Accordingly, it has been estimated that in India at least 1.2 million people suffer from active epilepsy due to NCC. The NCC form of Solitary Cystericercus Granuloma has been identified as the most common form of Neurocysticercosis prevalent in India with up to 60 percent of patients suffering from this type (this was first identified in 1989. The prevalence of this disease differs state-wise but in the southern Indian states, it was found to be extremely and moderately endemic. Conclusively this disease is prevalent in virtually all Indian states. Another interesting and rare finding is that over $95 \%$ of Indian NCC patients are vegetarians with this disease affecting only a limited proportion of pork eaters[4].

\section{Pathogenesis}

The Taenia solium's life cycle is a complex one that includes two hosts that can either be definitive or intermediate. Infected with the adult form of T.solium i.e. the tapeworm, humans become a definitive host. This happens when the individual eats undercooked pork which can still contain viable T.solium cysts. After ingestion, the cysticerci travel to the small intestines and bind to the mucosal wall of the intestine and grows to a length of around 1-8 meters. The patient may remain asymptomatic during this time or may show a few signs and symptoms. In human feces, terminal proglottids are released which may contain about 50,000 viable cysticerci. Pigs then become intermediate hosts if they eat anything infected with these ova and the same life cycle in pigs continues. If the ovum matures in the pigs, it penetrates the wall of the intestines and circulates in the blood, and is embedded in the muscles; they grow into the muscle larval type. When they ingest this meat, the cycle persists in humans' too. Throughout the process of cysticercus, where T.solium is larval, both humans and pigs will serve as intermediate hosts in which T. solium eggs are infected. Most of the time, the eggs enter the human body by consuming infected food or water. The ovum traverses the stomach, being partly digested, and releasing oncospheres. Such oncospheres cross the intestines and enter the bloodstream and pass through the walls. The oncospheres can then lodge in any tissue which includes the heart, retina, skeletal muscles, and brain. Usually, the oncospheres display a proclivity to the brain and settle there. The oncosphere's development in the brain is divided into four stages, as the signs and symptoms seen during the oncospheres presentation differ from stage to stage. During the first stage of infestation, one to two weeks after lodging in the brain by the oncospheres, it extends to form an edematous lesion and proceeds to grow and form a protoscolex with an enclosed bladder wall. In this case, a diagnosis will usually rarely be made. In this step, patients typically remain asymptomatic but those with severe infections may experience uncontrolled seizures and progressive dementia.

Moving to the next level, about 2 months after ova ingestion, the cysticerci cellulose matures and the cyst has a protoscolex surrounded by a clear-flowing bladder. The living cyst only causes a slight inflammatory reaction and this protects the cyst against the immune system of the host. The patient remains asymptomatic until the cysts remain viable (about 2-10 years +) until numbers are huge. Neuroimaging such as MRIS performed at this stage reveals noncontrasting cysts without adjacent edema, suggesting that these cysts do not usually develop from this point and later beyond their mature size. The mature cysticercus dies during stage 3 of the disease, i.e. about 2-10 years later; the transparent fluid becomes thicker and opaquer. It leads to hyaline degeneration and mineralization. The $\mathrm{C}$. cellulosae antigens begin to leak at this point; resulting in an extreme inflammatory response (this immune response is both humoral and cell-mediated with fibroblasts forming a capsule-like structure around the cyst). It takes between 6-18 months for the cyst to degenerate. In this process, it has also been found that oncospheres normally lodge in the brain 
parenchyma, but in 10-15\% of patients, they lodge in ventricles or meninges and do not become standard cysts. The cysticercus lodges in the subarachnoid space at times, and can extend up to $5 \mathrm{cms}$. These giant cysticerci produce neurological focal signs and heightened intracranial pressure. Chronic inflammation in the subarachnoid space may cause traverse artery vasculitis, leading to brain stem thrombosis, and some can develop focal neurological symptoms due to vasculitis from a stroke. Arachnoiditis can lead to obstructive CSF pathways and cause hydrocephalus that causes patients with dementia, depression, and stupor to develop an altered mental state. Throughout this stage, patients also display signs and symptoms with the main one being seizures that may be focal or widespread. The cyst dies spontaneously or from the anthelminthic treatment in this process when it comes to the 4 th and final level. The wall breaks, and the cyst transforms into a granular tissue with a dense collagen capsule. The granulomas calcify as time passes, thus the CT scans do not display any lesions. The patient is asymptomatic since the cyst is already dead, but some patients have recurrent seizures [5].

\section{Clinical manifestations}

Signs and symptoms of this disease differ between individuals because of the pleomorphic aspect of Neurocysticercosis. The severity of the symptoms varies in various individuals based on these factors, depending on the number of cysts found within the CNS to its size and location. These are important determinants in assessing NCC-related symptoms, too. The symptoms can be categorized as neurological, a common type of manifestation; and as symptoms of extra neurology. The extra neurological signs are typically rare and occur in subcutaneous and intramuscular cysticercosis cases. Patients present with severe body swelling or muscle areas affected by it. In these patients, acute myopathy can rarely develop. There may also be involvement of the head, neck, and trunk in these forms but the preponderance of its cranial involvement is unknown yet. The most prominent signs are the neurological symptoms, with seizures as a classic symptom. Besides, the patient can also experience extreme headaches and ataxia. The location of the cysts plays a major role in determining the type of NCC and the symptoms are classified accordingly. The aforementioned signs are typically seen in parenchymal NCC patients. The patients may show obstructive hydrocephalus in extra-parenchymal NCC and may experience stroke if the cysts are in the subarachnoid region. Increased intra-crane pressure is an obvious symptom of a cyst found in the ventricles. Another variation of this type of NCC is the racemose NCC which is characterized by the accumulation of multiple cysts at the base of the brain resulting in the patient displaying signs of mental decline, coma, and even life-threatening complications in some cases. Symptoms for ocular involvement include diplopia and chronic eye pain. Such signs can also lead to the development of other conditions that can also be life-threatening if not treated properly. Based on the type of NCC and its associated symptoms the complications may be of any kind. Many of them are dysarthria, excessive eye movement palsy or paresis, hemiparesis or hemiplegia; which can be associated with stroke, hemisensory impairment, motion disturbances, hyper/hyporeflexia, gait disruption, and even death [6].

\section{Diagnosis}

Throughout the years, with the advent of new diagnostic technology, diagnostic strategies for neurocysticercosis have improved significantly, but with the current tools, successful diagnosis of NCC is still extremely difficult. It is still very difficult to distinguish between parenchymal and intraventricular cysticercosis with the available methods of neuroimaging, particularly in developing rural areas. In these cases, it is important to use diagnostic tests to determine the condition and to arrive at a correct diagnosis. Del Brutto et.al's (2001) proposed diagnostic criteria incorporate different aspects of experience, clinical symptoms, immunological tests, and neuroimaging to create a well-formed framework for NCC diagnosis. The first and foremost step would be to collect the patient's history which includes medical history, medication, and travel. Travel history plays a significant role in deciding whether the patient has traveled to an endemic area and contracted the disease. A physical examination must be done but the signs and symptoms caused by NCC are not typically clear and it can be very difficult to differentiate between NCC forms based on these symptoms only. The most common signs are hallucinations, focal findings, fever, and hydrocephalus, or intracranial hypertension. The less common signs are non-specific, focal neurology findings. The explanation for that could be due to differences in lesion forms and mechanisms. CNS neuroimaging is very important to confirm the diagnosis and to assess the type of mechanism for the disorder, the emergency steps to be taken, and the correct choice of treatments. Which of the different methods of neuroimaging is the strongest is a topic to ask. MRI is much superior to CT scanning to visualize the brain structures and anatomy as well as the characteristics of the cysts. However, to detect calcifications, it has been found in many scenarios that a CT scan was superior to MRI. The best documented and most useful serological diagnostic method is a serum Western blot that employs a specific fraction of T. solium cysts. This is a very specific test to identify exposure and/or disease and also to confirm the diagnosis. However, in patients with the minimal disease with no specific symptoms, it lacks sensitivity and cannot be used alone for an accurate diagnosis. In recent time's antigen detection tests that use monoclonal antibodies (developed against the closely related T. saginata) have also been developed in an ELISA capture method. They are unique to detect pathogens that are 
currently viable. In the CSF, the antigen concentration is greater than in the serum. Hence the rates are highly associated with the extent of involvement. Some studies indicate that effective or correct results can be obtained from both ELISA and western blotting [7].

\section{Treatment}

A collection of recommendations for the clinical treatment of patients affected by Neurocysticercosis is prepared by the panel of the American Infectious Disease Society (IDSA) and the American Society of Tropical Medicine and Hygiene (ASTMH) [8]. Such recommendations include various strategies for the treatment and management of affected patients as well as for the different forms of NCC, such as intraventricular, subarachnoid, and others. The treatment options mentioned below are taken from the Guidelines released. The patients may be treated based on their intracranial pressure for the treatment of viable intraparenchymal NCC. For patients with untreated hydrocephalus, it is only important to control high intracranial pressure. In those with diffuse cerebral edema, diagnosis can be done using antiinflammatory drugs such as corticosteroids, and the hydrocephalus diagnosis requires a surgical method. The use of antiparasitic drugs is advised in all patients with VPN in the absence of elevated intracranial pressure. Here, the choice of treatment depends upon the number of viable cysts. For patients with one to two viable parenchymal cysts, it is recommended to administer Albendazole at a dose of $15 \mathrm{mg} / \mathrm{kg} /$ day divided into two daily doses for 10-14 days with food (maximum dose of $1,200 \mathrm{mg} /$ day) as a monotherapy. Careful monitoring is to be done on the second and fifth days of anti-parasitic therapy as there is usually an exacerbation of neurological symptoms which is attributed to local inflammation due to the death of the larvae. For this reason, both Albendazole and Praziquantel are generally given simultaneously along with steroids to control the edema and also intracranial hypertension that may occur from the therapy. Albendazole (15 mg / kg / day) in combination with Praziquantel (50 mg / kg / day) is administered 10-14 days with food. For parenchymal cystic lesions that remain for 6 months even after treatment, it is suggested that retreatment with anti-parasitic therapy be performed after the end of the initial therapy course and is necessary for such patients. Clinical prognosis with antiepileptic medications, anti-parasitic therapy, and corticosteroids is a must for patients with multiple enhancing lesions and convulsions. The anthelminthic therapy of choice is the administration of albendazole $(15 \mathrm{mg} / \mathrm{kg} /$ day in twice-daily doses up to $1-2$ weeks) with a meal. For patients with Solitary Cercus Granuloma most prevalent in India, it is recommended that they are treated before anti-parasitic therapy with corticosteroid therapy. In patients with calcified parenchymal lesions, symptomatic treatment alone is advised rather than the use of antiparasitic medications, as they are not viable. Corticosteroids are recommended to not be regularly used in patients with isolated CPN and perilesional edema. In very rare cases, such as in patients with refractory epilepsy along with CPN, it is strongly recommended that seizure foci be assessed for surgical removal. Intraocular cysticerci should be treated with surgical removal rather than with anti-parasitic medications, as in these situations there are often risks such as loss of vision by using anti-parasitics. Corticosteroids are also used to decrease neurological symptoms such as cerebral edema due to parasite death, $\&$ are used in the primary treatment of chronic arachnoiditis cysticercosis. A reduction of cerebral edema requires up to $32 \mathrm{mg}$ of Dexamethasone a day. Administration of adjunctive corticosteroid therapy should begin and is strongly recommended, before anti-parasitic drugs. Dexamethasone is the most effective regimen at doses of between 4.5 and $12 \mathrm{mg} /$ day. When long-term steroid therapy is needed, prednisone at $1 \mathrm{mg} / \mathrm{kg} /$ day can replace dexamethasone [9]. Mannitol is also used for acute intracranial hypertension that is due to Neurocysticercosis, at doses of $2 \mathrm{~g} / \mathrm{kg} /$ day. Antiepileptic medications are prescribed in all seizure-positive NCC patients. Seizures secondary to Neurocysticercosis usually respond well to antiepileptic first-line drugs. Single antiepileptic medication (AED) also controls seizures well. The length of antiepileptic treatment remains unclear but it does not depend on the type of seizure presented or any other recurrence risk factors, such as age at onset and number of pre-diagnostic seizures. Recurrence of seizures after removal of AED is associated with several lesions before beginning cysticidal therapy and with persistence or calcification of lesions after therapy. In patients with few seizures before anti-parasitic therapy or in patients showing cystic lesion resolution on imaging tests without seizures for 24 consecutive months, then tapering off and avoiding antiepileptic therapy can be considered [10]. Neuroendoscopy surgical procedures, open surgery, shunt placement is important in treating complicated stages of the disease. Neuroendoscopy is useful for the removal of quickly approaching cysts, which also cause symptoms. It can avoid excessive use of anthelminthic and corticosteroids. It is proposed that medical therapy and often a shunt requirement to control hydrocephalus may be effectively employed [11], according to a treatment sequence of 4th ventricular cysts lodged there.

\section{Prevention}

Neurocysticercosis is a widespread infection seen mainly in developing countries where conditions such as inadequate sanitation, insufficient disposal of human waste are seen prominently in favor of T. solium growth and transmission. But the disease can be eradicated with effective preventive steps. Preventing the first step is to wash your hands with 
soap and warm water after using the toilet, changing diapers, and before handling food. Teaching children, the importance of washing hands to avoid infection is extremely necessary. WHO \& CDC recommends that appropriate food and water health practices be adopted when traveling in endemic countries. Although in endemic regions it is recommended to drink only bottled or boiled water or carbonated beverages (1 minute) in cans or bottles, and also to filter unhealthy water through an "absolute 1 micron or less" filter and dissolve iodine tablets in filtered water ("absolute 1-micron" filters can be found in camping and outdoor supplies stores) [12]. It has become a growing concern among food handlers that there is a lack of hygiene practices where the practice of daily hand-washing is necessary but it is ignored. More light should be shed on how Neurocysticercosis can be prevented by taking steps to disrupt T's life cycle. Fortunately, pigs can now be treated with anti-parasite drugs and vaccinated against T. solium that prevents transmission of the parasite to humans. WHO is collaborating with all countries on tailoring enhanced monitoring methods to strengthen the care of the patients affected [13].

\section{Conclusion}

Being listed by the WHO as a neglected tropical disease, Neurocysticercosis is a widespread public health issue found mainly in developing countries at varied frequencies. But even, developed countries are not spared from the parasite thanks to the influx of refugees from infectious areas. The disease, its propagation \& prevention are issues that need to be put up to a public forum and should be addressed thoroughly to identify strategies and solutions for minimizing and preventing the spread of this infectious disease around the world. Countries facing deprivation and the lack of medical services and prognostics are very vulnerable to this disease's ill effects. These populations do not have access to appropriate neuroimaging or immunological testing services, making it very difficult for effective diagnosis. To maintain control over the spread of the disease, strategies must be implemented to avoid human-pig or vice-versa. Treatment is typically symptomatic and is meant to monitor any symptom. Pharmacotherapy involves anthelminthic and antiepileptic medications to treat the classic NCC symptoms. Drugs currently available yield results that may be negligible. The location of the cysts, or even the stage of the infection, may often become an obstacle or even extremely dangerous in obtaining beneficial results from the treatment. This can be attributed to the lack of adequate diagnostic facilities which hinder the selection of the necessary or even the provision of suitable individualized care. To improve and pave the way for new diagnostic approaches and treatment targets for treating NCC, further science and clinical work are needed. Also, well-defined research should be performed to determine different factors that may be hereditary or even pathological, which may or may be associated with the disorder. An integral consequence of this work would be that all elements of the disease that were previously overlooked or ignored must be taken into consideration when developing new treatment recommendations or when formulating ways of managing and preventing the disease. It is therefore very important to share with the public even the simplest of information to raise awareness of this issue. To ensure NCC is treated as a differential diagnosis in patients with seizures or serious headaches, a hard-and-fast rule must be made. Shedding light on these overlooked issues would help build interest in researchers working for improvement in developed countries ' conditions. Prevention and socio-economic growth are, as always, essential to removing NCC from the face of the earth.

\section{Compliance with ethical standards}

\section{Acknowledgments}

We acknowledge Dr. S Ponnusanakar for his support and guidance.

\section{Disclosure of conflict of interest}

The authors declare none.

\section{References}

[1] Bouteille B Epidemiology of cysticercosis and neurocysticercosis. Médecine et Santé Tropicales. 2014; 24(4):367374.

[2] [Internet]. Scielo.br. 2020 [cited 7 July 2020]. Available from: http://www.scielo.br/pdf/anp/v68n2/22.pdf

[3] Nash T, Garcia H Diagnosis and treatment of neurocysticercosis. Nature Reviews Neurology. 2011; 7(10):584594.

[4] Goel D, Dhanai J, Agarwal A, Mehlotra V, Saxena V. Neurocysticercosis and its impact on crude prevalence rate of epilepsy in an Indian community. Neurology India. 2011; 59(1):37. 
[5] Journal "Morphologia" E Review Langman's Medical Embryology / 13th edition. - LWW, 2014. - 424 p. Morphologia. 2015; 9(2):107-108.

[6] Cysticercosis - NORD (National Organization for Rare Disorders) [Internet]. NORD (National Organization for Rare Disorders). 2020 [cited 1 June 2020]. Available from: https://rarediseases.org/rarediseases/cysticercosis/

[7] Garcia H, Nash T, Del Brutto O Clinical symptoms, diagnosis, and treatment of neurocysticercosis. The Lancet Neurology. 2014; 13(12):1202-1215.

[8] Garcia H, Nash T, Del Brutto O. Clinical symptoms, diagnosis, and treatment of neurocysticercosis. The Lancet Neurology. 2014; 13(12):1202-1215.

[9] White A, Coyle C, Rajshekhar V, Singh G, Hauser W, Mohanty A et al. Diagnosis and Treatment of Neurocysticercosis: 2017 Clinical Practice Guidelines by the Infectious Diseases Society of America (IDSA) and the American Society of Tropical Medicine and Hygiene (ASTMH). Clinical Infectious Diseases. 2018; 66(8):e49e75.

[10] Liu D, Ahmet A, Ward L, Krishnamoorthy P, Mandelcorn E, Leigh R et al. A practical guide to the monitoring and management of the complications of systemic corticosteroid therapy. Allergy, Asthma \& Clinical Immunology. 2013; 9(1):30.

[11] Burneo J, Cavazos J. Neurocysticercosis and Epilepsy. Epilepsy Currents. 2014; 14(2_suppl):23-28.

[12] CDC - Cysticercosis - Prevention \& Control [Internet]. Cdc.gov. 2020 [cited 1 June 2020]. Available from: https://www.cdc.gov/parasites/cysticercosis/prevent.html

[13] 10 facts about neurocysticercosis [Internet]. World Health Organization. 2020 [cited 1 June 2020]. Available from: https://www.who.int/features/factfiles/neurocysticercosis/en/ 